\title{
Implementing MOE/MAR: Balancing Project Management with Change Management
}

$\overline{\text { Stephanie Saull-McCaig, RoseAnn Pacheco, Pakizah Kozak, }}$

Susan Gauthier and Rebecca Hahn

\section{Introduction}

When Toronto-based University Health Network (UHN) initiated its Medication Order Entry/Medication Administration Record project (MOE/MAR) in 2001, it was well understood by the organization that this would be one of the largest change management initiatives undertaken. Being the largest academic hospital in Ontario and the eighth largest healthcare organization in Canada, a successfully implemented computerized physician order entry (CPOE) project in this complex environment would require rigorous project management, significant clinical involvement and a well-developed change management program.

The extensive project management approach employed by UHN to support the MOE/MAR implementation will be discussed in this paper. The major phases of the MOE/MAR project are described from the perspective of the project team to highlight key activities undertaken, decisions made and challenges faced. Next, an analysis of the project management process is provided along with reflections of the approach taken and challenges encountered. Consistent with UHN's methodology to incorporate knowledge gained from the early phases of the MOE/MAR project into future implementations, we hope to provide insight to other organizations considering CPOE initiatives.

\section{The Complexity of MOE/MAR Requires Years to Complete}

The UHN Project Management Team (hereafter the "Project Team") was comprised of dedicated project management, information technology and Medical Informatics staff from the hospital's Shared Information Management Services (SIMS) department. Collaborating extensively with the hospital's Nursing and Pharmacy Informatics departments, these people were collectively responsible for delivery of the MOE/ MAR implementation.

The MOE/MAR project phases required over five years to complete, from the initial exploration phase to the end of the enterprise rollout. The majority of this time was invested up front on the System Design/Build phase and subsequent Pilot implementation to address numerous technical complexities. The majority of the clinical workflow implications of the MOE/MAR project were revealed in preparation for "go-lives" of each of the clusters (i.e., 
Figure 1. MOE/MAR major project phases

\begin{tabular}{|c|c|c|c|c|c|}
\hline $\begin{array}{l}\text { Initial } \\
\text { exploration } \\
\text { Begin evaluation } \\
\text { of project } \\
\text { requirements \& } \\
\text { engage vendors }\end{array}$ & $\begin{array}{l}\begin{array}{l}\text { System } \\
\text { design/build }\end{array} \\
\text { System build \& } \\
\text { rollout plan } \\
\text { Internal \& vendor } \\
\text { collaboration } \\
\text { Interface } \\
\text { development } \\
\text { between HIS* \& } \\
\text { RxS** }^{*}\end{array}$ & $\begin{array}{l}\text { Pilot } \\
\text { One general } \\
\text { internal } \\
\text { medicine ward } \\
\text { In parallel with } \\
\text { existing } \\
\text { paper-based } \\
\text { system }\end{array}$ & $\begin{array}{l}\text { 1st. Go-Live Part A } \\
\text { (Cluster \#1) } \\
\text { All GIM*** \& emergency } \\
\text { admitted patients } \\
\text { Conducted extensive } \\
\text { testing in controlled } \\
\text { production environment } \\
\text { prior to go-live } \\
\text { Order entry only to focus } \\
\text { solely on physicians }\end{array}$ & $\begin{array}{l}\text { 1st. Go-Live } \\
\text { (Cluster \#1) } \\
\text { All GIM \& } \\
\text { emergency } \\
\text { admitted } \\
\text { patients } \\
\text { eMar only to } \\
\text { focus solely } \\
\text { on nurses }\end{array}$ & $\begin{array}{l}\text { Enterprise } \\
\text { Rollout } \\
7 \text { additional } \\
\text { clusters } \\
\text { MOE/MAR } \\
\text { simultaneously } \\
\text { Overlapped rollouts } \\
\text { \& planning for } \\
\text { optimum timing \& } \\
\text { resource utilization }\end{array}$ \\
\hline
\end{tabular}

a grouping of clinically similar or closely related Nursing Units where MOE/MAR was implemented together). The major project phases are depicted in Figure 1.

\section{Investigation and System Build Require Longer- Than-Planned Early Phase}

The Project Team spent two years working with its Hospital Information System (HIS) and pharmacy (Rx) system vendors to prepare for the first pilot of the new combined MOE/MAR system. A considerable amount of effort and time was spent customizing the medication order entry functionality within the HIS and developing the technical Health Level 7 (HL7) interfaces to the Rx system to meet the needs of these stakeholder groups. Although UHN had extensive experience with building the interface between its HIS and other departmental applications, this particular phase of work proved more complex than any other interface project in the past, thus requiring longer time than originally anticipated.

Although considerable effort and investment had already gone into the technical design and build work, it was not until the final "green light" was received from UHN's Executive Management in 2003 that the subsequent steps of implementation planning could proceed.

\section{Pilot Test Marks First Project Milestone with Clinicians}

An initial pilot was conducted in February 2003. The test was confined to one General Internal Medicine (GIM) ward at the Toronto General Hospital (TGH). Electronic MOE and MAR capabilities were activated for physicians and nurses within that group. The pilot was designed to test whether the new system would allow physicians to order medications electronically. As a precaution against unforeseen problems, it was decided that both systems - the existing paper-based process and the new online system - would run simultaneously. Although this doubled workload for clinicians - physicians not only had to enter orders on-line, for example, but also had to write them out on paper - this approach helped with problem identification, and eliminated risks to patients.

Unfortunately, the pilot test had to be terminated earlier than expected due to significant technical performance problems not encountered during the system-build phase of the project. Although the system had worked well in a development environment, system delays emerged when MOE/MAR was subjected to far greater usage than originally anticipated. When this happened, the clinicians reverted back to the old paper process with no clinical impact. For some, however, a temporary black cloud had emerged over MOE/MAR.

Despite the dissatisfaction among some of the clinical staff, the silver lining for the Project Team was that the pilot was only terminated due to system performance problems that were considered solvable. It must be remembered that the pilot was intended to uncover possible weaknesses; in this regard it was a success.

The organization maintained its resolve to continue with the implementation after the performance issues were addressed. "It wasn't just a case of the staff resisting change," according to one MOE/MAR Project Manager. "We looked closely at the concerns they were having with the system and found them to be valid. The pilot really opened our eyes about the extent to which the system needed to be tested prior to going into the live environment. In the production environment there were many 
people entering, changing, holding and discontinuing orders. Realistic system load, from network traffic combined with variations and changes to real medication orders and administration routes and times, was a reality that the Project Team was not able to duplicate in the development environment."

Disappointingly, the technical performance issues took nearly a year to resolve before the Project Team could even begin to revamp its testing procedures prior to any further go-lives. Despite this setback, the Project Team worked closely with the system vendors, UHN's information technology experts and the key clinical teams to rebuild the system and to develop better ways to test the system under production-like conditions.

\section{Getting Ready for the Full Implementation: Rebuilding Internal and External Confidence}

In addition to working closely with the clinical staff in Internal Medicine to improve the system following the initial pilot, the Project Team put significant effort toward rebuilding the confidence of clinical staff with MOE/MAR to ensure they were committed to using the system again. For example, the key physicians within the group were identified and one-on-one meetings were conducted with them to increase their familiarity with the system and gain their support for its functionality and workflow.

Acutely aware of how the system's design had worked well in the development environment (albeit collapsing under the stress of a real production environment), the Project Team opted to conduct a short "proof of concept" test prior to the next full implementation. The system was set up once again on the Internal Medicine ward in the live production environment, but with usage restricted to members of the Project Team. Several weeks were spent extensively testing the system by using fake patients and fake medication orders, all within the context of Internal Medicine's real operational policies and workflow.

"We were very happy we chose to do it this way," recalled one Project Manager, "because we found a number of technical issues similar to those experienced during the first pilot that we were able to fix and re-test in a controlled environment." Once again it was back to the drawing board in terms of working with the system vendors (although this time around, for a far shorter period). Once the problems were resolved, the Project Team was confident that the system was ready for its first real MOE/MAR go-live.

\section{Strong Teamwork Marks First Go-Live}

In June 2004, the new system was brought up to full production mode for two GIM Units, plus the admitted patients in the Emergency department at TGH. This doubled the number of patients compared to the initial pilot. The Project Team chose to go live with this group first because it would provide the most stringent test of MOE/MAR. The Emergency and GIM

\section{... key physicians within the group were identified and one-on-one meetings were conducted with them to increase their familiarity with the system and gain their support for its functionality and workflow.}

departments provided some of the most complex set of services in the hospital. These services cared for a broad spectrum of patients and illnesses with the most combinations of required medications than in any other clinical service. The GIM service uses a team-based approach to patient care, involving physicians, nurses and pharmacists working collaboratively within the units. The Project Team's decision was to pick the services with the most complex set of medication orders as the starting point for MOE/MAR with the expectation that subsequent clusters would be incrementally easier.

As the first cluster, both Project Team and clinical staff faced especially substantial skill and knowledge development challenges. As such, clinical staff in the Emergency and GIM departments had to contribute significant amounts of time to ensure that all potential workflow scenarios were anticipated and addressed prior to implementation.

This first MOE/MAR deployment in the Emergency department and GIM service at TGH was referred to as "Cluster \#1." The Project Team was careful not to call it another "pilot" in order to avoid the (incorrect) message that this was another test. During this initial cluster, the Project Team and participating clinical staff gained much knowledge about how to improve subsequent clusters, particularly controlled production testing. It took approximately four months for Cluster \#1 on the GIM and Emergency Units at the TGH site to be considered by clinicians to be "running smoothly." Although considered a success by hospital senior management, getting to Cluster \#1 had taken significantly more time than anyone originally anticipated. The Project Team's morale was waning in anticipation of the massive amount of work ahead and the Senior Project Manager and other Project Managers knew that streamlining the implementation process in the future would be critical to maintaining the team's momentum.

\section{Rolling Out MOE/MAR across the Organization}

During the Cluster \#1 implementation, the Project Team and senior clinical leadership of the hospital planned the sequence of implementations across the rest of the enterprise. The remaining clinical services were divided into nine additional clusters based on similarity in patient flow, staff workflow, complexity of medication orders and geographic proximity. As well, clinical leadership advised that MOE/MAR in surgical services should 
Table 1. MOE/MAR Clusters

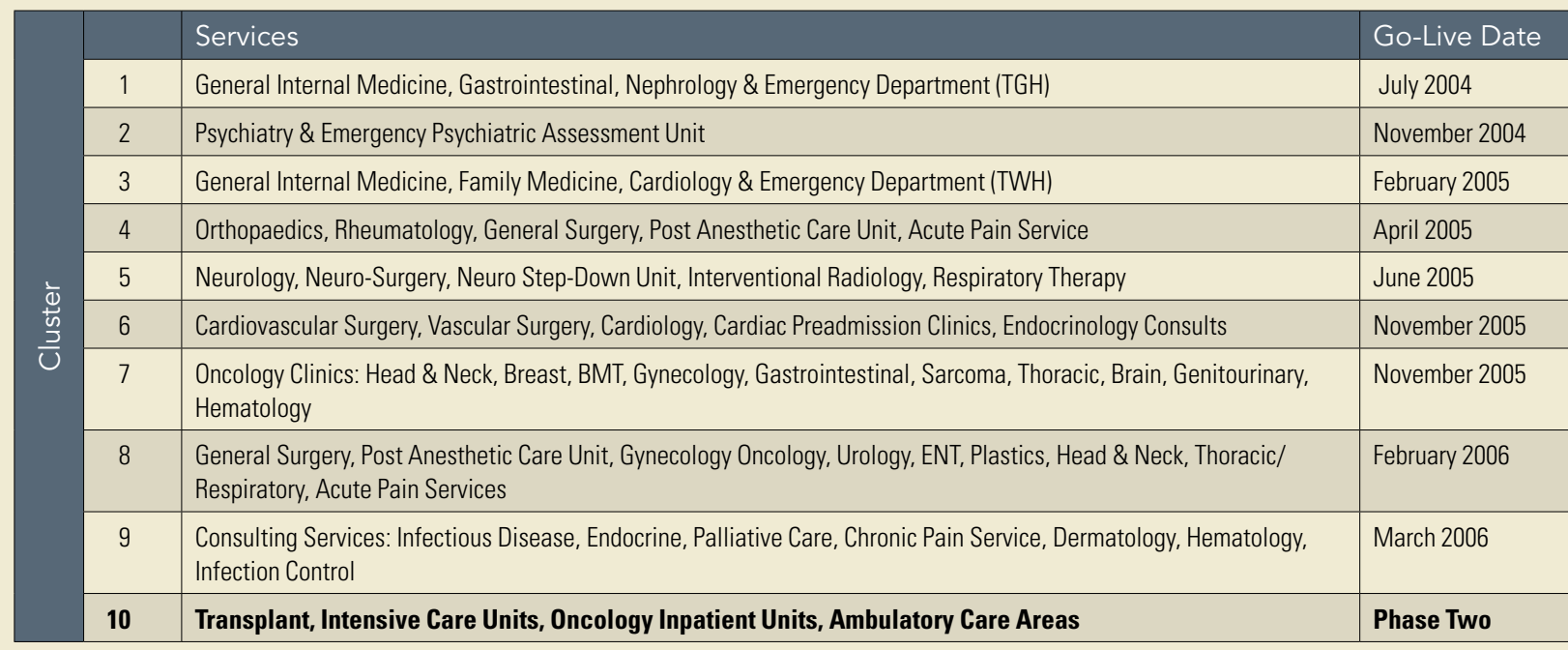

go late in the sequence to allow more time to assess clinical workflow redesign requirements in these exceptionally complex service areas. Table 1 lists all 10 clusters with their actual MOE/ MAR go-live dates.

The Psychiatry Units at both TGH and Toronto Western Hospital (TWH), which are a more defined and smaller group of patients and clinicians, were selected as Cluster \#2. The rationale for putting Psychiatry second was to select a service that had a relatively high likelihood of success. The Project Team wanted to quickly gain credibility with the clinicians. As well, the Senior Project Manager felt the Project Team needed a boost in confidence. Cluster \#2 proved to be a "quick win" compared to Cluster \#1, and the Psychiatry clinicians and the Project Team were able to celebrate a second success in a comparatively short time frame. Another benefit of gaining momentum in this manner was that it created a "halo effect" around the project, which secured more support from decision-makers and stakeholders throughout the organization.

Cluster \#3, which included Internal Medicine at TWH, also went relatively smoothly because the Project Team had developed experience and credibility through its efforts with Internal Medicine at TGH during Cluster \#1.

With each additional cluster, the Project Team gained confidence and credibility; as well, the project as a whole gained momentum. Thus, on it went, cluster by cluster, over the course of approximately 20 months, during which the Project Team successfully implemented MOE/MAR in most inpatient areas across TWH and TGH and all ambulatory services at $\mathrm{PMH}$ using the same, but increasingly more streamlined and regimented deployment process for each cluster.
By the end of March 2006, MOE/MAR had been implemented and was fully operational in the first nine clusters (eight and nine were combined at the end). To date, however, UHN has yet to implement MOE/MAR within the Intensive Care Units, Transplant Unit or Chemotherapy Inpatient Units due to the highly complex medication and workflow needs not fully supported by the current technology. To our knowledge, few hospitals in North America have successfully implemented MOE/MAR within these environments for the same reasons experienced at UHN. The Project Team continues to explore required enhancements with our system vendors and assess clinical workflow redesign requirements to accommodate these areas.

\section{Project Management Tactics to Support Clusters}

Project management rigour was essential to ensure a wellplanned and disciplined MOE/MAR implementation. UHN project managers and technical staff applied Project Management Institute (PMI - see www.pmi.org) principles and processes as well as Information Technology Information Library (ITIL) best practices for change management. To further support the MOE/MAR project, the Project Team also employed two major tactics that were key to keeping the project on an aggressive rollout schedule: (1) using a fluid project team organization structure combined with (2) a well-ordered and consistent cluster implementation cycle.

\section{Project Team Organization Structure}

During the rollout, the Project Team was organized into a number of smaller change management teams. These smaller sub-teams could work in parallel on as many as three clusters 
Figure 2. MOE/MAR project structure and roles

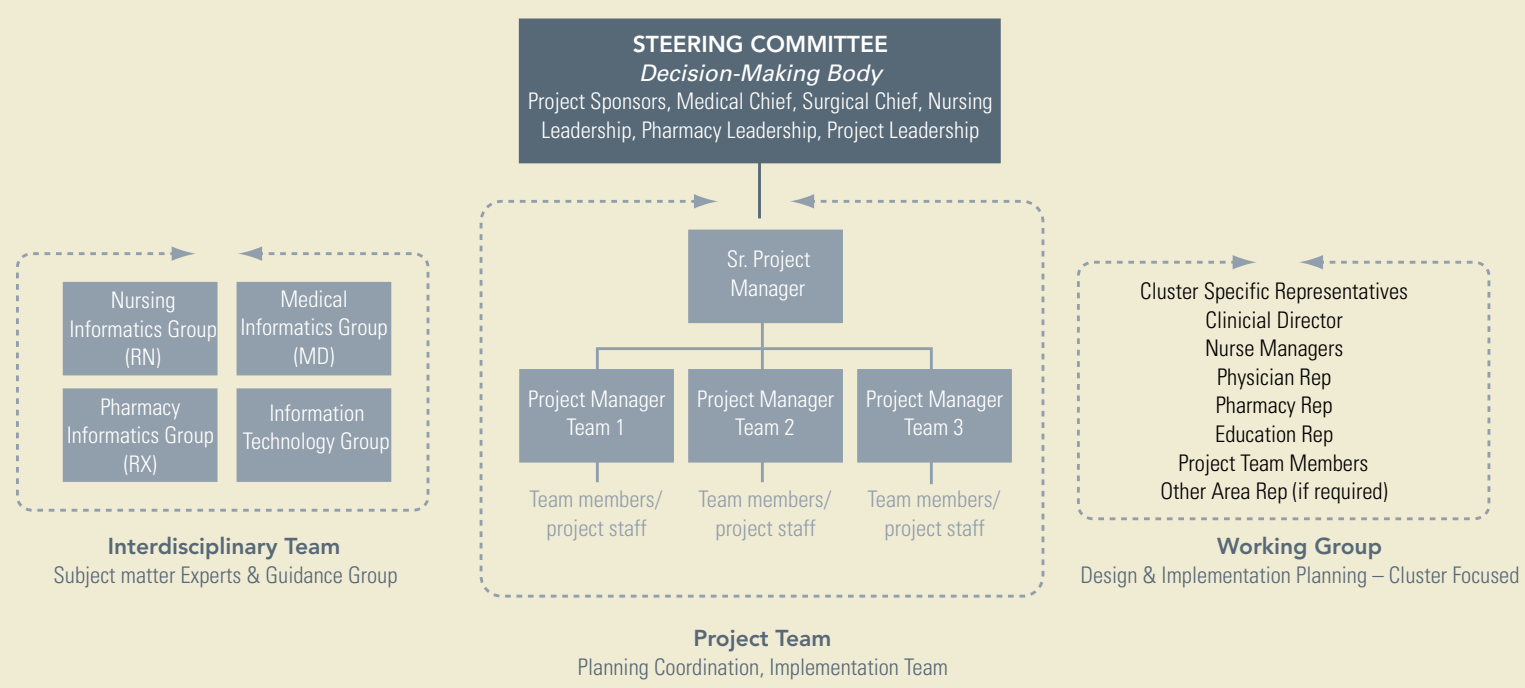

at a time even though they were each at a different stage of the implementation cycle. This approach enabled the Project Team to follow an aggressive rollout schedule and leverage expertise developed across clusters (see Figure 2).

The Project Team was able to rapidly hone its skills in training, support, relationship building, negotiation, issues management and change management by continuously problem-solving across multiple clusters. The Project Team also implemented a formal process to reflect on its successes and failures as it went along, allowing the learning curve to be greatly shortened for each subsequent cluster.

\section{MOE/MAR Cluster Implementation Cycle}

One of the most important tactics to keep the project momentum high was to utilize a consistent set of activities with each cluster. As the Project Team began to develop an understanding of the appropriate sequence of implementation steps, it began to codify its "methodology" to be rolled out in subsequent clusters. Figure 3 depicts this project methodology.

Each cluster's kick-off meeting was crucial in helping key clinical stakeholders understand why UHN was implementing the MOE/MAR project. In addition to highlighting the benefits of on-line medication order entry (to their patients, to UHN and ultimately to them) the meetings addressed expectations about what the system would and would not do. We also requested the commitment required from the clinical leadership to make the process successful.

Subsequently, weekly clinician engagement meetings were held with the clinical teams (Pharmacy, Nursing, Physicians) to undertake the crucial change management activities that would prepare each area for the MOE/MAR implementation. These activities included workflow analysis and redesign, medication order set development, downtime preparation, device analysis and status updates. The level of attendance and participation by stakeholders in these meetings made for a reliable barometer of how successful the implementation of MOE/MAR would be.

The environmental device assessment involved an analysis of each physical area to determine the number and types of point-of-care devices (e.g., electronic whiteboards, mobile medication carts, desktop computers, laptops) that would be required. Managing stakeholder expectations was crucial during this activity since many clinicians considered this an opportunity to "go shopping." This was the Project Team's opportunity to distinguish between clinician wants and requirements.

Next, a team of industrial engineers conducted pre-implementation time and motion studies to measure patient safety indicators in the paper-based medication environment. These metrics were later compared to post-implementation metrics representing the electronic environment in order to quantify and understand the impact MOE/MAR had on patient safety. The results of these studies are presented in "The Benefits of the MOE/MAR Implementation: A Quantitative Approach," (see p. 77 in this issue).

A team of Database Analysts then built service-specific screens with order sets within the HIS database. Both technical and clinical members of the Project Team tested these order sets extensively to ensure their accuracy and appropriateness.

Back order entry, which was conducted the weekend before 


\section{Figure 3. MOE/MAR project methodology}

\section{Three to six month effort}

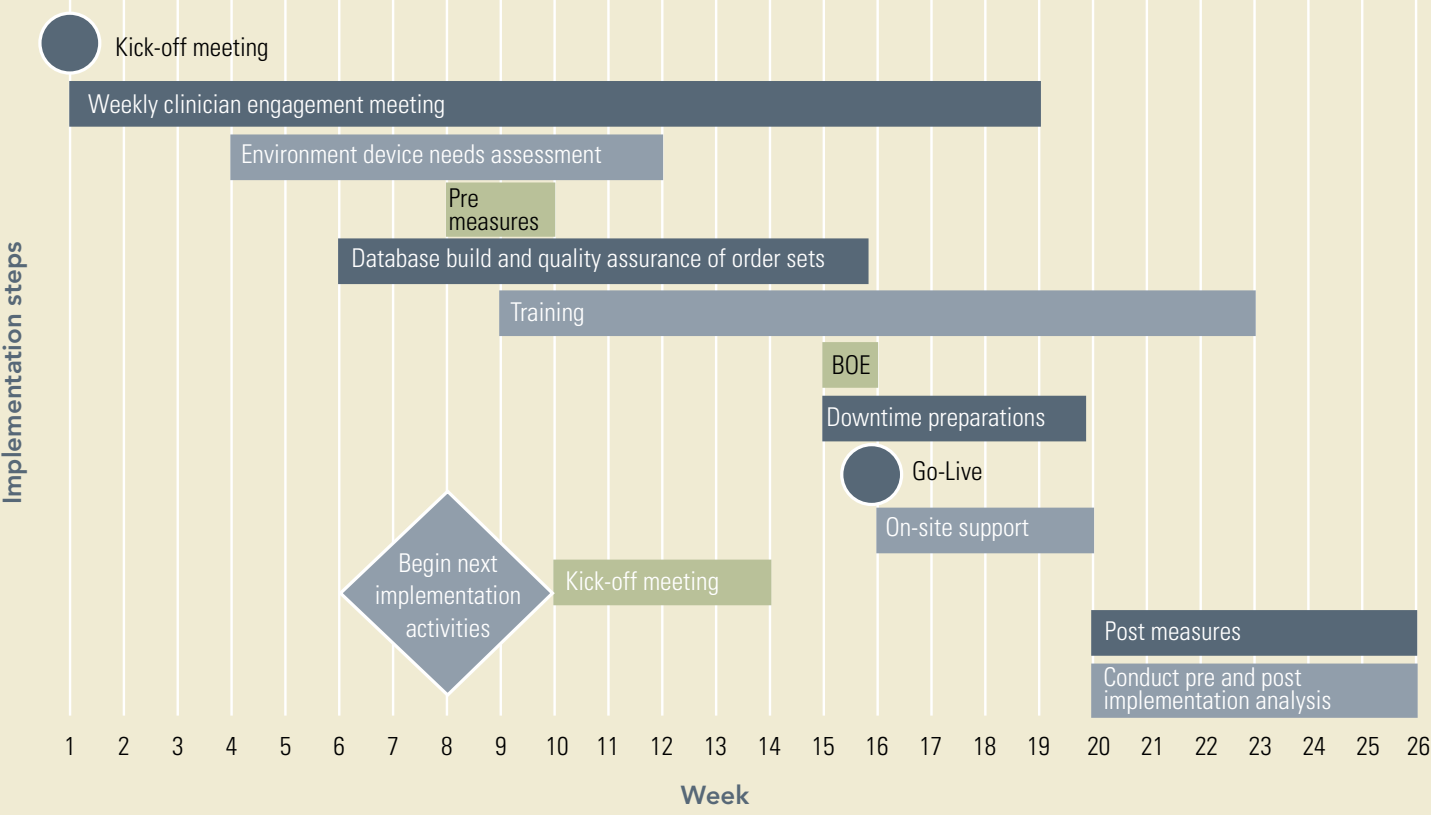

each go-live, was an extremely detailed and labour-intensive process requiring pharmacists to enter all paper-based medication orders for each admitted patient in a particular cluster. A second pharmacist and a nurse verified each order to ensure accuracy.

Downtime preparation included installing a designated downtime computer and printer in each area. We also conducted in-service training on each ward to ensure clinicians knew how to activate emergency downtime procedures in the event of a system outage. As well, the go-live included four weeks of onsite, 24/7 support following each MOE/MAR implementation, during which daily auditing activities were conducted to ensure the new system was being utilized efficiently and safely. Then, and to this day, we believe this was crucial to MOE/MAR's success.

The use of a consistent project management methodology helped the Project Team plan more effectively than if they had used a customized approach for each cluster. It also helped to manage expectations among the clinical teams about their roles when it came time for them to participate in MOE/MAR. Supported by parallel, sub-Project Teams, we could maintain multiple clusters working simultaneously, thus enabling the planned enterprise implementation at a rate of one cluster per four months on average.

\section{Reflections on Implementations}

Through its formal learning process, the MOE/MAR Project Team compiled volumes of lessons throughout the entire project. Again, since there were few models of successful MOE/MAR implementations to look to in other hospitals, most of these lessons came from trial and error. This section highlights the most important reflections gleaned from our UHN experience. We acknowledge that in many respects UHN is not a typical hospital. However, we believe that the following reflections can be generalized to any organization contemplating a major CPOE implementation, as they primarily relate to general change management principles.

\section{Avoid Re-inventing the Wheel}

Given the scope and complexity of UHN's MOE/MAR initiative, the Project Team sought external project planning and implementation guidance. We did extensive research and sought advice from other hospitals (especially those using the same HIS) that were undertaking similar CPOE initiatives. Most of these hospitals are in the United States.

"We spoke to a number of institutions via phone, and even conducted some site visits," reported a Manager of Information Management. "Although we were able to take a few pieces from here and there, we found that no one was able to provide a 
single, complete project management or implementation model that was suitable for our use."

To further strengthen UHN's ability to manage such a complex project, it hired external consultants who had clinical informatics or project management expertise in other healthcare institutions. These consultants were able to suggest valuable ideas about project planning and support. The consultants also validated the Project and Technical teams' change management practices. An independent pharmacy consultant and a nursing consultant were hired to strengthen the clinical support capabilities of the two respective informatics groups. While the consultants were not involved throughout the entire project, they were extremely valuable at the beginning of the Project to fill in skill gaps on the evolving Project Team.

\section{Workflow Redesign Is an Iterative Approach}

For physicians and nurses, administering medications is at the very core of how they treat their patients. Implementing MOE/MAR not only impacted how they ordered, procured, administered and documented medications, but also impacted how they communicated with each other. Removing the physician order sheets and paper MARs removed the tools that had been used to convey information between clinicians; thus it was necessary for them to develop alternative means of communication. "Thinking that MOE/MAR is just a medication IT solution would be a serious mistake," reflected a Director of the Project Team. "It's a change management initiative focusing on medication, but it also has a broad impact on the way people in the hospital work."

Redesigning clinical workflow for the electronic environment first required a complete understanding of how clinicians worked in the paper environment. Clinicians were able to describe the way things worked in the paper environment, but it was more difficult to obtain agreement on how things should work in the new electronic world. Often, when processes were redesigned for the electronic environment, they would initially turn out to be impractical or inefficient.

The workflow redesign became an iterative process requiring many attempts to reach a desirable end state. The use of mock scenarios walking through the major workflows and understanding when, where and what information was needed - helped identify holes in proposed processes and generated new and better solutions. Upon reflection, the Project Team could have conducted more (and better staged) mock scenarios. Had it done so, the resulting workflow would have better illustrated undesirable occurrences that often did not surface until golive. When this happened, the Project Team had to correct them in a reactive mode. Indeed, one of the greatest challenges for the Project Team was identifying what they did not know.

\section{Expect to Uncover Pre-existing Clinical Practice Issues}

Several inconsistent clinical practice standards and policy issues surfaced during the project as a consequence of conducting in-depth workflow analysis and automating manual processes. Although many of these predated MOE/MAR, the project nevertheless often brought these issues "out into the light." For example, the majority of patients

\section{Lessons Learned}

1. Avoid re-inventing the wheel - Seek outside assistance if possible and learn from others' mistakes first to avoid wasting time and energy up front.

\section{Workflow redesign is an iterative} approach - An on-line medication management system must be designed around clinical users and their workflow. It is important to review, resolve and standardize, wherever possible, all relevant clinical workflow policies and practices before designing the system.

3. Expect to uncover pre-existing clinical practice issues - Clinical practice issues and inconsistencies will be exposed when assessing workflow for MOE/MAR. Although they may predate the project, they will need to be resolved to carry on with MOE/MAR implementation.

4. Credible champions are key to managing change - Select project "champions" who recognize the benefits of on-line medication order entry and medication administration; who have practical and comprehensive knowledge of the daily clinical processes within their services; who are respected by their peers as credible leaders; and who can challenge peers and others when needed.

\section{Different services have very different} needs - Understand the differences in culture between various hospital services (Medicine, Surgery, Anesthesia, etc.), and make sure the project timelines and workflow design are adjusted appropriately to accommodate those differences.

6. Expect politically motivated interference - Do not underestimate the time, effort and resources required in handling project and organizational politics. Project team members should have strong facilitation and negotiation skills to manage sensitive situations.

7. Continuous communication sets the tone - Do not "sugar-coat" messages; direct and open communication styles work best when giving tough messages. Also, be prepared to use a wide variety of communication vehicles to ensure that people at all levels receive the key messages.

8. Proactively manage expectations - MOE/ MAR is a clinical transformation initiative, not just an IT project. The Project Team needs to be able to effectively negotiate with clinical leadership on "needs" versus "wants" in order to gain support for implementation.

9. Take time to celebrate your successes - Appreciate that a little recognition goes a long way to maintaining the enthusiasm and momentum needed. 
prepped for surgery are given an order for an antibiotic to be administered "on call" to the operating room by the anesthetist. The antibiotic is typically sent along with the patient from the ward, physically attached to the patient's chart. During the MOE/MAR workflow analysis for these medications, it was discovered that often anesthetists typically disposed of the antibiotics that accompanied the patient from the ward and later administered an alternate antibiotic. In this case, the project had exposed a previously existing problem. Although this practice did not have a direct impact on how the MOE/ MAR system would operate in the environment, pressure was put on the Project Team to address this issue prior to the implementation in the surgical areas. Realizing this practice issue did not require to be resolved to proceed but would still remain an issue in the post-implementation environment, the Project Team continued with the rollout and highlighted the ongoing issue to existing clinical committees for resolution. MOE/MAR also enabled pre-existing policies to be better enforced than in the previous paper world, where it was difficult to monitor non-compliance. For example, under federal policy, Advanced Practice Nurses (APNs) are prohibited from ordering controlled substances, including narcotics. However, in analyzing their workflow, it was discovered that APNs were previously not prevented from ordering controlled substances (such as Ativan and several types of narcotics). With MOE/MAR, however, the controlled substances policy is automatically enforced through role restriction in the HIS, making it impossible for unauthorized personnel to order restricted medications.

\section{It was quickly discovered that the most} effective champions were those who: recognized the benefits of on-line medication order entry and medication administration; had practical and comprehensive knowledge of the daily clinical processes within their services; were respected by their peers as credible leaders; and, most importantly, were comfortable in challenging peers and others when needed.

UHN did not have a forum for these types of issues to be identified prior to MOE/MAR. As such, the Project Team often took on many of them directly, even though they were clinical practice issues rather than directly attributed to the MOE/MAR implementation. In response to MOE/MAR having highlighted a number of these types of clinical practice issues, UHN initi- ated a new corporate "Clinical Best Practice and Information Technology" committee to be a regular forum where clinical leadership could debate and resolve issues in a timely fashion.

\section{Credible Champions Are Key to Managing Change}

At the beginning, the Project Team struggled to find the right mix of people to serve as MOE/MAR "champions" or people that would informally lead other clinicians to adopt MOE/MAR. The Project Team initially turned to clinicians who were "techies" (i.e., those comfortable using new software systems). These individuals tended to be younger clinicians rather than seasoned veterans of their clinical service. Although highly enthusiastic, their junior status hampered their ability to challenge more senior clinicians, and thus diminished their success convincing others of the value of the MOE/MAR initiative.

To facilitate the MOE/MAR rollout, meetings were held with senior leadership within each cluster to identify Physician, Pharmacy and Nursing "champions" to assist the change management team in preparation for the go-live. Working closely with the Project Team on as much as a daily basis leading up to the go-live, UHN's champions supported the workflow analysis efforts and provided specific information needed by the Project Team for building the system, redesigning workflow and preparing to deliver training. The champions also helped with decision-making and with communicating and promoting the project to their colleagues. It was quickly discovered that the most effective champions were those who: recognized the benefits of on-line medication order entry and medication administration; had practical and comprehensive knowledge of the daily clinical processes within their services; were respected by their peers as credible leaders; and, most importantly, were comfortable in challenging peers and others when needed.

Since the time commitment of these champions was significant, the Project Team conducted introductory meetings with them as early as three to four months ahead of go-live - six months ahead in the case of a complex cluster. Most often, these champions - having been identified by the most senior clinical leadership in UHN - willingly engaged with the Project Team immediately. However, escalation to senior leadership was sometimes necessary to "encourage" less enthusiastic individuals to work more closely with the Project Team.

The Project Team never made any final decisions without first getting the cluster champions to sign off (literally) on project plans, workflows and screen designs. This process demonstrated the significance of the champions' role in MOE/ MAR and to their units. From the initial introductory meetings through to go-live, meetings between MOE/MAR Project Team members and the cluster champions were held weekly, without fail, to plan for implementation. After go-live, these meetings continued on a daily basis through the end of each cluster's four-week support period. 


\section{Education, Training and Support}

Due to the large number of staff and the complexity of MOE/MAR, it was determined that training would be best achieved in a classroom setting. The instructional design of the courses - delivered as a suite of on-line tutorials, later complemented by on-line documentation - was done with support from an Education Working Group established for the MOE/MAR project, consisting of representatives from Education, the Project Team, Computer User Support Program and Nursing Informatics. Physician input was also sought during the development of the physician courses.

"This group, which met regularly throughout the MOE/MAR project to review and provide input on education agendas, content and issues, proved to be invaluable to the ultimate success of our education and training efforts," notes the Manager of SIMS Education.

A seven-hour classroom course on MOE/MAR was initially recommended for nurses, while physicians would attend a two-hour course. However, for staffing and financial reasons - it would simply cost too much to take nurses out of service for a full day - the classroom training for nurses was reduced to four hours in length. After MOE/MAR was deployed in the first two clusters, it became apparent that the four-hour classroom courses were not adequate to cover all the training that nurses required, and nurses and nurse managers began asking for longer training sessions. A new five-step education process developed by the Education Working Group provided staff with the training they needed (see accompanying diagram).

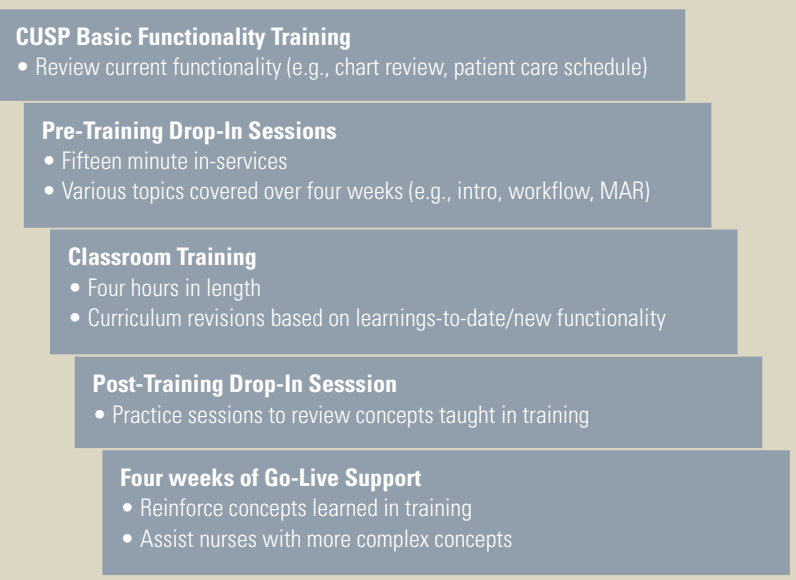

\section{Basic Functionality Testing}

A number of nurses coming to MOE/MAR classroom training benefited from some basic computer and EMR training. Staff from UHN's Computer User Support Program (CUSP) worked with nurse managers in each unit to identify nurses needing assistance, and then delivered basic computer and EMR training through a series of informal, one-on-one sessions conducted in the units.

\section{Pre-Training Sessions}

A series of 15-minute drop-in MOE/MAR demonstration sessions on each Nursing Unit were included in the training process to relieve apprehension over the new system. This helped increase comfort and knowledge levels before staff went to the classroom, making them better prepared to receive the actual MOE/MAR system training.

\section{Classroom Training}

Early in the implementation, the project educators found that classroom time for nurses was being consumed by workflow and logistics questions such as how MOE/MAR would work on their units, how the new pointof-care carts would be used, and so on. While all the questions were valid, they nevertheless took up too much classroom time; sometimes the questions had the result of derailing the classroom effort altogether. To address this problem, the Education Working Group members created a "day in the life" series of photos shot on a ward where nurses were already using MOE/MAR. Through this presentation, nurses got a good idea of what MOE/MAR would look like. Frequently Asked Question (FAO) sheets were also created and distributed in the classes, providing information on the post-training sessions and the go-live support as well as contact numbers for the Project Team. With workflow-type questions and issues minimized, the four-hour classroom period could be used solely to train nurses how to actually use MOE/MAR. The accompanying chart summarizes the scale of the classroom effort.

After the initial design of the classroom training, the Education Working Group played a key ongoing role as a feedback and curriculum update mechanism. The group provided a forum for discussing class content priorities and helped to ensure that the curriculum was updated as system functionality changed over the course of the full MOE/MAR rollout.

\begin{tabular}{|c|c|c|c|c|}
\hline Group & $\begin{array}{l}\text { Length of } \\
\text { Course }\end{array}$ & $\begin{array}{l}\text { Average } \\
\text { Class } \\
\text { Size }\end{array}$ & $\begin{array}{l}\text { Number } \\
\text { of } \\
\text { Classes }\end{array}$ & $\begin{array}{l}\text { Number } \\
\text { Trained }\end{array}$ \\
\hline Physicians - MOE/MAR only & 2 hours & 3 & 423 & 1,281 \\
\hline $\begin{array}{l}\text { Physicians - new to UHN + } \\
\text { MOE/MAR }\end{array}$ & 4 hours & 5 & 129 & 587 \\
\hline Nurses & 4 hours & 5 & 434 & 2,226 \\
\hline Pharmacists & 4 hours & 5 & 31 & 138 \\
\hline Pharmacy technicians & .5 hours & 9 & 2 & 18 \\
\hline Respiratory technicians & 2 hours & 4 & 17 & 66 \\
\hline Support staff & $\begin{array}{l}3 / 4 / 6 \\
\text { hours }\end{array}$ & 5 & 19 & 101 \\
\hline Total & & & 1,055 & 4,417 \\
\hline \multicolumn{5}{|c|}{$\begin{array}{l}\text { Notes: } \\
\text { - Physicians new to UHN attended a four-hour class (instead of the two-hour class) } \\
\text { covering basic HIS functionality as well as MOE/MAR functionality. } \\
\text { - The Physician courses included Staff Physicians, Residents, Fellows, Medical Students } \\
\text { and Advanced Practice Nurses. } \\
\text { - The Nurse groups were comprised of separate courses for Nurses, Nurse Managers, } \\
\text { Registered Practical Nurses and Nursing Students. }\end{array}$} \\
\hline
\end{tabular}

\section{Post-Training Sessions}

To ensure maximum information retention, classroom training should ideally take place no more than three weeks prior to go-live. Due to the large number of nurses that had to be trained, however, and because they could only be taken off the wards a few at a time, the training for each cluster had to start as much as six or seven weeks ahead of the go-live, by which time much of what they learned could easily have been forgotten. To keep the classroom training information fresh in the nurses' minds leading up to the go-live, the Project Team conducted practice sessions on the units that focused on functional areas identified by the nurse managers as being key to that unit. By reinforcing the classroom learning, these post-classroom sessions proved to be most effective in ensuring a smooth go-live, but only when attendance was mandatory; otherwise, few nurses attended. 


\section{Post-Go-Live Support}

The purpose of this phase of the education was to reinforce the training the nurses, physicians and pharmacists had received during the first four steps of the process and to help them resolve problems they were encountering while actually using MOE/MAR to manage their medication management activities.

Each go-live was followed by a four-week period during which the Project Team provided 24/7 on-site (i.e., right on the unit) support to give clinicians immediate face-to-face assistance as they got comfortable with the system and to help them solve technical or workflow problems as they arose. It was initially thought that four weeks of support might be too long, but it turned out to be necessary. Although the bulk of the questions came during the first couple of weeks, the extra time was needed to cover the unit staff's changing rotation.

During this phase of the education, the project team was expanded through the addition of other staff members from Nursing Informatics, Pharmacy and the Information Management departments to ensure a high level of clinical, technical and project management support capability. Support team members continually roamed within the unit, wearing red lab coats that read "SIMS Support" to make it easy for unit staff to pick them out on the busy floors.

After the Go-Live ... Knowledgeable Support Was Key

The questions asked and issues raised were all logged and tracked by the Project Team. Daily meetings between the Project Team and the clinical leadership were conducted during this four-week period to review each day's activities, discuss policy concerns and go through the list of outstanding issues (see diagram below for overview of types of questions received during the four-week support cycle). Resolving issues as quickly as possible showed clinicians that the Project Team was serious about their concerns, thus helping to build stronger relationships and credibility with clinicians in the units. The key to the success of these meetings was that they included the right people with the right authority, so that final decisions could be made and the project could move forward.

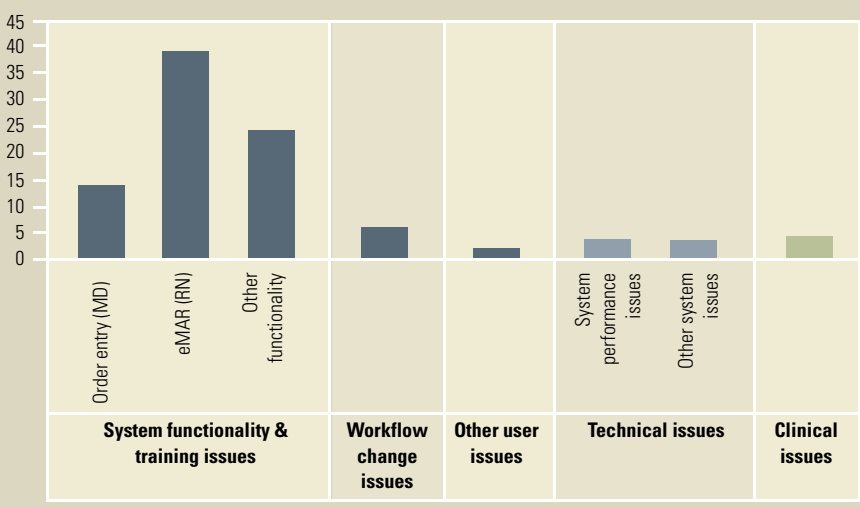

Over time, the Project Team was able to determine when it could taper the support staff on the units over the four-week support period while maintaining an optimal level of support for clinical staff. This was done in consultation with cluster champions, unit managers and other clinical staff on the unit, as each unit was considered unique in its needs for post go-live support.

\section{Different Services Have Very Different Needs}

Apart from the need to customize the MOE/MAR system to meet the unique functional and workflow requirements of each cluster, significant cultural and workflow differences were also encountered between various services such as Medicine, Surgery and Anesthesia and specialty services such as the Acute Pain Service. Different services had different needs, thereby presenting different challenges to the Project Team. The impact these differences had on our go-live schedule was initially underestimated.

\section{General Internal Medicine}

GIM at UHN is a very collaborative environment where nurses, physicians and pharmacists typically round together. In terms of working with the MOE/MAR Project Team, the physicians showed themselves to be problem-solvers willing to try things out and to compromise. In addition, the staff were willing to incorporate MOE/MAR into their teaching. This helped new residents rotating through the service learn how to use the new system.

\section{Surgical Services}

Surgeons and surgical residents are primarily focused on performing surgery. Since most of their time is spent in the operating room, surgeons are usually off the unit for the majority of the day; thus their ordering practices are distinctly different from those in Medicine. Surgeons typically order medications when they do rounds each morning, visiting each of their patients and adjusting their meds as required. After rounds, however, there are often very few surgeons left on the ward to help the nurses solve any problems that might arise with those orders.

\section{Acute Pain Service}

The Acute Pain Service deals with the pain needs of both medical and surgical patients throughout the hospital. As such, this group required that their orders be uniquely identifiable from all others. They also required their own queues for identifying and tracking their patients, who could be anywhere in the hospital. Due to the high-risk nature of the drugs that they order, their ordering practices are meticulous and extremely focused on patient safety. As a result, they had high expectations for sophisticated clinical decision support capabilities in the system, including drug-drug interaction checking and duplicate order alerts. 


\section{Anesthesia}

Because anesthetists operate at such a fast pace and are always under great pressure to keep patients moving through the operating rooms, anesthetists have little time to enter postop medication orders. With a shortage of staff and with surgery cases often scheduled back to back, anesthetists were highly concerned about the impact on delaying subsequent cases due to the time it might take to enter medication orders.

\section{Expect Politically Motivated Interference}

The MOE/MAR project was politicized from the start. Politics existed between middle management and hospital executives; between the three clinical disciplines (Physicians, Nursing and Pharmacy); between the various hospital services; between the individual units within a single cluster.

For all of its meticulous project plans and Gantt charts to estimate time to complete each of the standard activities in a given cluster, the Project Team significantly underestimated the amount of time required to address the politicking throughout the project. Many MOE/MAR Project Managers soon became expert facilitators, negotiating among various groups to bring various political issues to rest. These facilitators often got mired in the middle of issues that had little to do with the technical aspects of the project, but as rollout leaders they were expected to address these issues.

For example, Project Team members were often challenged with questions about the benefits of the initiative and why UHN was investing in such a large project. Because many staff perceived the project as likely to increase their workloads, they resisted the change. Project Team members were often criticized for being too pushy or for making decisions without having all the information. In spite of this resistance, however, the Project Team had to keep the project moving forward. For nearly half of the 20-month project, the Project Team was consumed with relationship development, political adversity and helping intended users appreciate how MOE/MAR could help them improve patient care and safety.

\section{Continuous Communication Sets the Tone}

At the beginning of the project, the Project Team tried to "sugar- 
coat" the communications regarding the impact the new system would have on the staff. In retrospect, it was obvious that its failure to be fully open with senior management (in other words, shielding senior management from some of the implementation problems) was a mistake, as it risked confidence in the Project Team's ability to deliver. There was also considerable initial nervousness about sharing too much too soon, so initial communications were very targeted rather than broadbased. The Project Team's initial strategy was to wait until it was absolutely sure it was going to be successful before initiating communications.

After the lessons of Cluster \#1, the Project Team started using a variety of mass-communication vehicles - e-mail, newsletters and UHN's intranet - to inform the clinical population about the benefits the system could deliver as well as the potential impact it could have on them as individuals. As part of their effort to build face-to-face relationships across the organization, Project Team members took advantage of every opportunity to present the MOE/MAR story to as many groups as possible. Key messages in later communications came to be more up front about the need for the substantial knowledge and skill development that would be needed to use MOE/MAR.

A number of project management and reporting tools were used on an ongoing basis to keep hospital staff informed of the project's progress. Biweekly Status Reports were sent out to a massive distribution list, providing an update on project milestones, issues and risks. A project Report Card used green, yellow and red indicators to score how each unit was progressing in terms of measures such as the percentage of physicians that were placing orders directly into the new system (see Figure 4). Interestingly, this report card led to an informal competition between the units based on their respective CPOE rates, which pressured less compliant units to improve.

\section{Proactively Manage Expectations}

A big challenge was to continuously manage the staff's expectations. Because UHN was an early adopter of this new technology, the Project Team expected that difficulties would emerge. Open and honest communications helped staff stay focused on the bigger picture goals, making it easier for them to accept that new changes to the system could take multiple years to implement.

Another constant challenge for the Project Team was staying focused on the project scope and distinguishing between legitimate user needs versus wants. Virtually every service within the hospital had a "wish list" of things they wanted that were beyond the scope of what the MOE/MAR project could afford or deliver (mainly in the areas of system functionality and device requests). Although most of these were revealed during the initial workflow assessments, additional requests surfaced during golive and the subsequent support phase. Rather than taking these wish lists at face value, the Project Team had to work closely with cluster champions to identify the true priorities.

\section{Take Time to Celebrate Your Successes}

$\mathrm{MOE} / \mathrm{MAR}$ was a long and difficult project involving commitment and considerable effort from many people. As each cluster was successfully deployed, an executive site visit was conducted to bring senior hospital leadership from across the organization, including the CEO, to congratulate the unit and thank frontline staff for their hard work. A site-wide celebration was also held after the rollout of MOE/MAR was completed in each hospital, which was well attended by front-line clinical staff. While not initially budgeted, these celebrations were well worth the expense as they became important milestones to signal the hospital's appreciation for the staff's contribution. They also helped maintain the enthusiasm and momentum as deployment continued in other clusters.

\section{Conclusion}

In what most feel was the most challenging, yet most rewarding project they had ever been involved with, the Project Team spent a great deal of time and effort ensuring that the MOE/ MAR deployment went as smoothly as possible. Throughout all phases of the MOE/MAR project, each cluster challenged the Project Team to develop new approaches and more efficient project management methods of engaging clinicians and leadership in the change process. Although UHN clinicians are now able to access the full functionality of MOE/MAR, the work continues. MOE/MAR is a significant step to realize UHN's goals of improved patient safety.

\section{About the Authors}

Stephanie Saull-McCaig is the Director of Acute Care Information Management, Shared Information Management Services (SIMS) at University Health Network. Contact: Stephanie.Saull-Mccaig@uhn.on.ca

RoseAnn Pacheco, RN, BASc, MA, is the Manager of Clinical Documentation and Record Services, Shared Information Management Services (SIMS) at University Health Network.

Pakizah Kozak, MBA, is Manager of Information Management, Shared Information Management Services (SIMS) at University Health Network.

Susan Gauthier, MHSc, is a Manager of Education, Shared Information Management Services (SIMS) at University Health Network.

Rebecca Hahn, BA, PMP, is a Project Manager in Shared Information Management Services (SIMS) at University Health Network. 
Commentary

MOE/MAR Project Management: A (Well-Informed) Bird's Eye View

Denise Zarn

A $s$ an outsider looking in, I can confidently say that UHN has done a spectacular job implementing MOE/MAR. The University Health Network (UHN) has long viewed information management (IM) as important to its mission to provide exemplary patient care and innovative research and teaching. Consequently, for the past five years UHN has asked my company to conduct an annual review of the hospital's IM strategy and related implementations. So again this year, as leader of the Accenture review team, I was privileged to get a first-hand look at UHN's progress. Our review included in-depth interviews with executives and clinicians as well as staff focus groups drawn from across the organization that gave the MOE/MAR (order entry) project high praise. We also applied other measures, including a survey that was responded to by nearly 500 physicians, nursing and allied health professionals.

\section{I also know from my own involvement in UHN's first order entry project nearly 20 years ago that implementing MOE/MAR has been no quick-fix, overnight task.}

I also know from my own involvement in UHN's first order entry project nearly 20 years ago that implementing MOE/ MAR has been no quick-fix, overnight task. When the hospital first embarked on its electronic patient record journey, it was thought it would be accomplished in three years. However, no one imagined then how difficult this project would be in the long run. In response, the organization and the staff in charge of the electronic patient record project (of which MOE/MAR is a significant portion) have consistently shown an unusual amount of organizational tenacity, paired with substantial investment to support its IM implementations - $4 \%$ of operating budget annually, compared with $1-2 \%$ at most other Canadian hospitals.

What's behind UHN's IM success to date? Rigorous project management expertise and achieving actual senior clinician sponsorship are two success factors that my team has witnessed over the past five years.

SIMS (UHN's shared information management services group) is expert at project management - and not just by adhering closely to good project management principles. Eighty percent of SIMS' project managers are certified by the Project Management Institute (PMI) and the rest are in progress for their certification. SIMS has nurses, physicians and other clinicians on staff who provide clinical expertise and credibility to its implementations. All SIMS project staff must complete rigorous user requirements, clinician-approved test and training plans and evaluations of every project to ensure that projects deliver on plan. When SIMS project staff put a project plan together, the Project Management Office enforces it aggressively.

\section{What's behind UHN's IM success to date? Rigorous project management expertise and achieving actual senior clinician sponsorship ...}

Because order entry has such significant clinical impact, SIMS very carefully and deliberately engaged senior clinicians alongside senior executive management to own the project. Although our review uncovered multiple occasions where obstinate clinicians could have derailed the entire MOE/MAR initiative, sponsors readily addressed issues head-on before they could undermine the project.

This year the release of our IM review coincided with final implementation of MOE/MAR's phase-one rollout. And how did people feel about it by then? It was very encouraging to find from our survey and focus groups that $73 \%$ of UHN clinicians understand the goals of UHN relating to information management and the electronic patient record, and that MOE/MAR was rated as the Number One information technology tool that has had the most positive impact on clinicians' work environment.

\section{About the Author}

Denise Zarn is a partner in the Health and Life Sciences Practice at Accenture, Inc. She has been involved in physician orderentry projects for over 20 years and has worked on advancing electronic patient records at the enterprise and regional levels both in Canada and the United States. 